\title{
Alles, was eine Wirkung hat, hat auch eine Nebenwirkung ...
}

\section{Christoph Bosshard}

Dr. med., Vizepräsident der FMH, Mitglied des Zentralvorstandes der FMH, Departementsverantwortlicher Daten, Demographie und Qualität / Schweizerische Akademie für Qualität in der Medizin SAOM

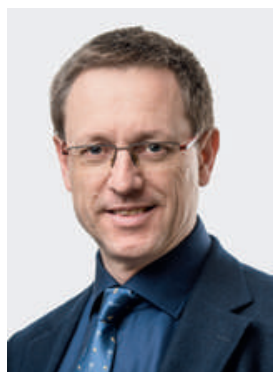

Dass neben der beabsichtigten Wirkung auch Nebenwirkungen auftreten können, wissen wir seit Paracelsus und wenden diese Erkenntnis in unserem ärztlichen Alltag an. Wir sind stets bestrebt, Nutzen und Risiken sowie Nebenwirkungen für unsere Patientinnen und Patienten abzuwägen und mit ihnen gemeinsam den für sie besten Weg zu finden. Dass die Ärzteschaft sich auch hierzu intensiv Gedanken macht, war schon mehrfach Gegenstand unserer Schweizerischen Ärztezeitung: Ich erinnere an Publikationen zu Shared Decision Making, Guidelines sowie Indikationsqualität.

Nutzen und Risiken einer Behandlung abwägen und gemeinsam mit den Patienten den für sie besten Weg finden ist zentraler Bestandteil der ärztlichen Tätigkeit.

Die Patienten, welche uns am meisten beschäftigen, sind konfrontiert mit vielschichtigen gesundheitlichen wie auch psychosozialen Herausforderungen. Ihre Situation ist dabei stets individuell. Patentrezepte wären für alle Beteiligten eine Erleichterung. Nur gibt es solche eben genau so wenig wie es die Standard-Patientin, den Standard-Patienten gibt.

Wie viel Medizin brauchen Patienten? Wie viel Gesundheitswesen benötigt unsere Gesellschaft? Die erste Frage können wir häufig erst nach der eigentlichen Patientenbehandlung diskutieren, und dies auch lediglich vor dem Hintergrund der getroffenen Entscheidungen und des begangenen Weges. Ob eine andere Entscheidung, ein anderer Weg besser gewesen wäre, bleibt im Einzelfall hypothetisch. Auf gesellschaftlicher Ebene beobachten wir eine zunehmende Medikalisierung von Fragen, welche eigentlich mutig andernorts zu diskutieren und zu entscheiden wären. Vielleicht beruht die Tendenz der Medikalisierung auch darauf, dass der Bürger seinem von ihm so gewollten Gesundheitssystem viel zutraut, erreicht die Schweiz hier doch in internationalen Vergleichen im- mer wieder Spitzenwerte! Der Drang nach Sicherheit ist eine ebenfalls nicht zu unterschätzende Kraft. Nur: Kann das System dies so alles leisten? Und welche Nebenwirkungen sind dabei auf System-Ebene zu erwarten? Es mag wenig erstaunen, dass bei einer guten Versorgung und Zugänglichkeit die Tendenz eher in Richtung Overuse tendiert, bei einer Mangel-Versorgung hingegen in Richtung Underuse.

Unser in dieser Ausgabe der Schweizerischen Ärztezeitung präsentiertes Grundlagenpapier (Seite 236) thematisiert primär die Overuse-Frage, wenngleich die anderen Aspekte nicht minder wichtig sind. Weitere Abgrenzungsfragen stehen in Form der Begriffe Minimal-, Maximal- sowie Optimalversorgung im Raum. Wo beginnt nun der Overuse, wo sind die Nebenwirkungen grösser als der Nutzen? Wie gestalten sich diese Überlegungen auf individueller und auf System-Ebene? Was ist die Solidargemeinschaft bereit, im Rahmen der Sozialversicherungen zu tragen? Wo finden lediglich Kosten-Verschiebungen statt, da der gesamtwirtschaftlich nachhaltige Blick fehlt, der Nutzen sich nicht da einstellt, wo die Kosten anfallen? Warum sprechen wir immer nur von Kosten und so selten von Nutzen?

\section{Over- und Underuse in der medizinischen} Versorgung sind letztlich auch Fragen von gesellschaftlicher Tragweite.

All diese Fragen müssen wir Ärztinnen und Ärzte uns stellen, um unsere Erfahrungen und Sichtweise einzubringen. Versorgungsfragen wie Over- und Underuse sind letztlich auch Themen von gesellschaftlicher Tragweite. Unser Grundlagenpapier soll dabei mithelfen, die Diskussion mit der aktuell zur Verfügung stehenden wissenschaftlichen Evidenz zu unterstützen und auch die diesbezüglichen Grenzen aufzuzeigen. Bitte unterstützen Sie Ihre FMH auch hier in Form Ihrer aktiven Mitarbeit in Ihren Basis-Organisationen und Fachgesellschaften! 